\title{
The Impact of Infostation Density on Vehicular Data Dissemination
}

\author{
Vinod Kone • Haitao Zheng • Antony Rowstron • \\ Ben Y. Zhao
}

Published online: 18 November 2010

(C) The Author(s) 2010. This article is published with open access at Springerlink.com

\begin{abstract}
Vehicle-to-Vehicle and Vehicle-to-Roadside communications are going to become an indispensable part of the modern day automotive experience. For people on the move, vehicular networks can provide critical network connectivity and access to real-time information. Infostations play a vital role in these networks by acting as gateways to the Internet and by extending network connectivity. In this context, an important question is "What is the minimum number of infostations that need to be deployed in an area in order to support vehicular applications?" Optimizing infostation density is vital to understanding and reducing the cost of deployment and management. In this paper, we examine the required infostation density in a highway scenario using different data dissemination models. We start from a simple analysis that captures the required density under idealized assumptions. These models are validated by an event-driven simulator. We then run detailed QualNet simulations on both controlled and realistic vehicular traces to observe the informa-
\end{abstract}

\footnotetext{
V. Kone $(\bowtie) \cdot$ H. Zheng · B. Y. Zhao

Department of Computer Science, University of California, Santa Barbara, Santa Barbara, CA 93106-5110, USA

e-mail:vinod@cs.ucsb.edu

H. Zheng

e-mail: htzheng@cs.ucsb.edu

B. Y. Zhao

e-mail: ravenben@cs.ucsb.edu
}

A. Rowstron

Microsoft Research, Cambridge, UK

e-mail: antr@microsoft.com tion density trends in practical environments, and consequently propose techniques to improve dissemination performance and reduce the required infostation density.

Keywords infostation density $\cdot$ vanet . vehicular networks $\cdot$ data dissemination

\section{Introduction}

Vehicular networking is becoming a reality. The current generation of GPS-based navigation and information devices already incorporate wireless connectivity. As they grow to support features such as streaming traffic data, streaming audio and location-aware content, it is important to understand how to provide infrastructure to efficiently support these devices. In addition, as application data grows to include more time sensitive data (e.g., free parking spaces, traffic flow), users must update devices more frequently. A major challenge has been how to get timely data to these devices.

The current generation of devices uses a number of different wireless technologies to achieve this, including leveraging cellular infrastructures or broadcastbased infrastructures such as satellite or FM sub-carrier bands. A cellular approach implies devices "pull" data from the network. Devices can use the cellular infrastructure by either building cellular capability into the device, e.g. GM's OnStar system, or by connecting to a mobile phone using BlueTooth, e.g. the TomTom One. An alternative is to use a broadcast medium that pushes data to devices, including satellite networks, e.g. Sirius, or FM sub-carrier bands, e.g. NAVTEQ. 
Pragmatically, both cellular and broadcast approaches face disadvantages. The broadcast medium requires significant investments in large-scale infrastructure such as a global satellite network. In contrast, a cellular approach relies on infrastructure owned by a telecom company that controls network access and therefore requires per-country negotiation. Furthermore, incompatible cellular networks partition the user base depending on region. While connections made through users' mobile phones are region-independent, it has the major disadvantage that different data plans control the volume of data that can be pulled to the device. This information needs to be exposed to the information device, or else it can be very expensive for the user!

Since cellular and broadcast approaches are less than ideal, we turn our attention to an alternative based on infostations (or access points) assisted with vehicle-tovehicle propagation. Infostations are attractive because they can be incrementally deployed by a variety of vendors with comparatively low upfront costs. Zero cost vehicle-to-vehicle communications can decrease the infostation density, thereby significantly reducing cost of deploying infostations. This leads us to the question: Will vehicle-to-vehicle distribution combined with intermittent connectivity to infostations provide a suitable data dissemination system for vehicular networks? In particular, could a network of WiFi access points, such as those owned by T-Mobile, McDonalds, or Starbucks be used to deliver data to vehicles? What density of infostations would be required? In this paper we attempt to understand the trade-offs and limitations of infostation-based data dissemination, as the first stage of answering these questions.

We achieve this by modeling two different approaches to infostation-assisted dissemination in a vehicular network: push and pull. In the push model, infostations act as a data carousel continuously transmitting data. Vehicles receive the data when in transmission range of infostations, and then propagate the data to other vehicles, using epidemic data dissemination to extend data transmission to vehicles beyond the range of infostations. In the pull model, each vehicle has a data set that it requires. It attempts to pull the information from infostations, using multi-hop routing if necessary. We assume that each infostation has access to all the requested content.

The main goal of our paper is to understand the minimum infostation density required to support different data dissemination applications. We choose to consider a highway scenario because this is one of the most practical deployment scenarios. We begin by building simple analytical models of the two infostation- based dissemination approaches without considering wireless propagation and contention. These abstract models allow us to study in more detail the impact of transmission range, data size, and data lifetime on each approach in an ideal scenario. We use a simple simulator to confirm the correctness of the models and to determine the impact of vehicle density. To further understand trade-offs such as the impact of wireless channel contention, we perform detailed simulation experiments using QualNet [19] with vehicular traces derived from real-world vehicular traces of a segment of a Californian highway.

For each dissemination scheme, we show that the lifetime of data is a dominating factor on required infostation density. Our QualNet simulations show that a number of additional factors play crucial roles in dissemination performance, including the selection of forwarding neighbors, knowledge of nearby vehicle locations, and pull request density in the pull model. We propose some initial techniques to address these challenges and focus on examining their significant impact on the overall required density of infostation. Finally, experiments on a trace derived from US101 highway provide interesting insights into the impact of the wireless vehicle density on the infostation density.

\section{Related work}

There is increasing interest in vehicular networking, with a number of small scale vehicular networking deployments [5, 14, 23]. However, due to the small scale of these deployed systems, most prior work has focused on evaluating particular strategies and dissemination models. Heavy emphasis has been put on pushbased dissemination [12, 13, 15, 25], and dissemination through vehicle-to-vehicle propagation $[6,20,21,24]$. Some recent work on practical systems $[1,5,9,14]$ hints at the feasibility of pull-based distribution.

Nadeem et al. [15] examine vehicle-to-vehicle dissemination using the push model. They compare two push model variants; flooding and dissemination. In the flooding model information is generated by cars and flooded through the vehicular network, which clearly has scalability issues. Therefore, they also consider a push variant where vehicles perform batching of local information received from neighbors before pushing it to their neighbors. In [15], the authors study the impact of varying the set of vehicles that propagate information, such as to those vehicles traveling in the same direction. In [20], the authors propose a delay 
constrained routing protocol to deliver vehicle generated messages to nearby infostations.

Lochert et al. [13] also examine the feasibility of information dissemination in city environments using a push model. They conclude that the potential for low density of equipped vehicles means some infostations are required, and evaluate where they should be placed in a city environment. They also conclude that networked infostations perform better than infostations that simply act as a repeater for information heard from other vehicles. Zhao et al. [25] also model and simulate push based dissemination using infostations. They model the impact of delay at intersections.

In [22], the authors propose a dissemination protocol for comfort applications based on segmentation of the road. Scalability through aggregation of information and intelligent broadcasts are achieved with the help of a digital road-map. Uichin et al. [10] propose a buysell based virtual market place on vehicular networks. Dissemination is through neighbors that come into contact with each other. This scheme suits better to urban environments than highway scenarios.

While prior works summarized in the above have examined the performance of vehicle data dissemination, little attention was given on the impact of infostation density. Yet it is the major factor that will affect the deployment of infostations and the performance of dissemination. Different from prior work, in this paper we focus on examining the impact of infostation density in highway scenarios, using two dissemination models.

\section{Abstract models}

In this section, we develop simple analytical models for the infostation density required to support data dissemination in vehicular networks. A complete and accurate model of vehicular data dissemination is highly complex, because it must capture the impact of a large number of factors ranging from physical transmission characteristics to data lifetimes. Therefore, we derive simple analytical models for push and pull dissemination schemes that capture their defining characteristics. The goal of our models is to understand the high level trends for infostation density and the impact of content settings, i.e. data size and lifetime.

Specifically, we make the following assumptions:

- We assume the width of the road is much smaller than the transmission range of infostations and vehicles. This assumption is true in general if infostations and vehicles use WiFi to communicate.
- We assume that vehicular density is high, and all vehicles travel in the same direction at a uniform velocity $V$. Note that $V$ is much lower than the signal propagation speed.

- We assume that the impact of transmission errors and MAC contention/schedule is abstracted into the bandwidth and range of transmissions between infostation and vehicles and among vehicles. This abstraction allows us to perform the analysis without being confined to any specific MAC and physical configuration.

Table 1 summarizes the parameters used in our analysis.

\subsection{The push model}

In the push model, the data dissemination range $D_{\text {info }}$ represents the maximum distance that the data $i$ can travel to at the end of $i$ 's lifetime. We can separate the range into two parts: $r$, the distance covered by a broadcast from the infostation; and $R$, the distance covered by the subsequent forwarding by vehicles (in one direction). As shown in Fig. 1, the maximum coverage is $r+2 R$.

We can determine the distance $r$ as:

$$
\begin{aligned}
r & =2\left(d / 2-\left(d-R_{\text {info }}\right)\right)=2 R_{\text {info }}-d \\
& =2 R_{\text {info }}-\frac{S}{B_{\text {info }}} V
\end{aligned}
$$

where $d=\frac{S}{B_{\text {info }}} V$.

Table 1 Parameters used in the analysis

\begin{tabular}{ll}
\hline $\begin{array}{c}\text { Infostation parameters } \\
B_{\text {info }}\end{array}$ & Bandwidth of an infostation \\
$R_{\text {info }}$ & Transmission range of an infostation \\
$D_{\text {info }}$ & Dissemination range-distance between two \\
& neighboring infostations, or $1 / D_{\text {info }}$ is the \\
& density of infostations \\
Vehicle parameters \\
$B_{\text {mobile }}$ & Bandwidth of a vehicle when communicating with \\
$R_{\text {mobile }}$ & Transmission range of a vehicle \\
$V$ & Velocity of a vehicle \\
$I_{\text {arrival }}$ & Inter-arrival interval of vehicles \\
Data parameters & Size of the data $i$ \\
$S$ & Lifetime of the data $i$ \\
$T$ & Size of the pull request \\
$S_{\text {pull }}$ &
\end{tabular}




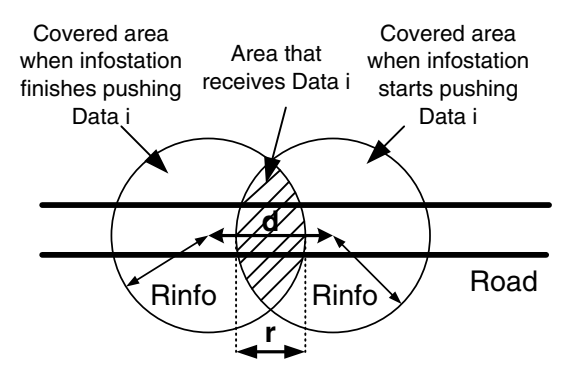

Fig. 1 Coverage of push. Left Coverage of infostation's broadcast. Nodes which obtain the complete content of data $i$ are only in the shaded area. $d=\frac{S}{B_{\text {info }}} V$. If $d \geq 2 R_{\text {info }}$ then the shaded area is NULL, i.e. no node can download the complete set of

Next, we derive the relative distance covered by vehicles forwarding data $i$ within its lifetime $T$, which we refer to as $R$.

$$
\begin{aligned}
& T=\underbrace{\left[\frac{R}{R_{\text {mobile }}}\right]}_{\text {\# of hops }} \cdot \underbrace{\frac{S}{B_{\text {mobile }}}}_{\text {delay per hop }}+\underbrace{\frac{S}{B_{\text {info }}}}_{\text {AP broadcast delay }} \\
& \Longrightarrow R=R_{\text {mobile }}\left\lfloor T \cdot \frac{B_{\text {mobile }}}{S}-\frac{B_{\text {mobile }}}{B_{\text {info }}}\right\rfloor
\end{aligned}
$$

Therefore, the total distance covered by the data dissemination within its life time $T$ is

$$
\begin{aligned}
D_{\text {info }}= & r+2 R \\
= & 2 R_{\text {info }}-\frac{S}{B_{\text {info }}} V \\
& +2 R_{\text {mobile }}\left\lfloor T \cdot \frac{B_{\text {mobile }}}{S}-\frac{B_{\text {mobile }}}{B_{\text {info }}}\right\rfloor
\end{aligned}
$$

When infostations and vehicles use the same radio configuration, $B_{\text {info }}=B_{\text {mobile }}$ and $R_{\text {info }}=R_{\text {mobile }}$, we have

$D_{\text {info }}^{\text {push }} \approx \frac{2 R_{\text {mobile }} B_{\text {mobile }} T}{S}-\frac{S V}{B_{\text {mobile }}}$.

\subsection{The pull model}

In this model, infostations never push data. Instead, any vehicle $n$ in need of data $i$ sends a request to nearby infostation to request it on-demand. ${ }^{1}$ We also assume that vehicles can download the locations of nearby infostations when they come into contact with an infostation. Therefore, the data dissemination process consists of two stages: (i) vehicles forwarding the pull request

\footnotetext{
${ }^{1}$ Note that when vehicles cache data, a node in between the infostation and requesting node $n$ can send the data to $n$. However, our analysis assumes that data $i$ is not available at any vehicles.
}

data $i$. Right Coverage of subsequent vehicle epidemic content distribution. Vehicles propagate data $i$ to both directions in multihops

to the nearest infostation; and (ii) the infostation delivering data $i$ to the source vehicle, if necessary using multi-hop forwarding by other vehicles. We will refer to the time spent in stage one as the delay of pull and the time in stage two as the delay of delivery. Figure 2 provides a graphic view of the stages.

The distance covered represents the farthest vehicle (from an infostation) which can pull and retrieve data $i$ within the data lifetime. That is, the sum of the delay of pull and the delay of delivery must not exceed the data lifetime. Clearly, whether a vehicle is to the left or right of the infostation matters in this case because the distance traveled by the pull request and its response is different.

First, for a vehicle at $R_{\text {right }}$ distance to the right of an infostation, the time required to retrieve data $i$ is:

$$
\begin{aligned}
T= & \underbrace{\left\lceil\frac{R_{\text {right }}-R_{\text {info }}}{R_{\text {mobile }}}\right\rceil \cdot \frac{S_{\text {pull }}}{B_{\text {mobile }}}+\frac{S_{\text {pull }}}{B_{\text {info }}}}_{\text {delay of pull }\left(t_{\text {right }}^{\text {pull }}\right)} \\
& +\underbrace{\left[\frac{R_{\text {right }}-R_{\text {info }}+V\left(t_{\text {right }}^{\text {pull }}+\frac{S}{B_{\text {info }}}\right)}{R_{\text {mobile }}}\right] \frac{S}{B_{\text {mobile }}}+\frac{S}{B_{\text {info }}}}_{\text {delay of delivery }}
\end{aligned}
$$

Similarly, for a node at distance $R_{\text {left }}$ to the left of an infostation, the time required to retrieve the data is

$$
\begin{aligned}
T= & \underbrace{\left\lceil\frac{R_{\text {left }}-R_{\text {info }}}{R_{\text {mobile }}}\right] \cdot \frac{S_{\text {pull }}}{B_{\text {mobile }}}+\frac{S_{\text {pull }}}{B_{\text {info }}}}_{\text {delay of pull }\left(\left(_{\text {left }}^{\text {pull }}\right)\right.} \\
& +\underbrace{\left[\frac{R_{\text {left }}-r_{\text {left }}-V\left(t_{\text {left }}^{\text {pull }}+\frac{S}{B_{\text {info }}}\right)}{R_{\text {mobile }}}\right] \frac{S}{B_{\text {mobile }}}+\frac{S}{B_{\text {info }}}}_{\text {delay of delivery }}
\end{aligned}
$$




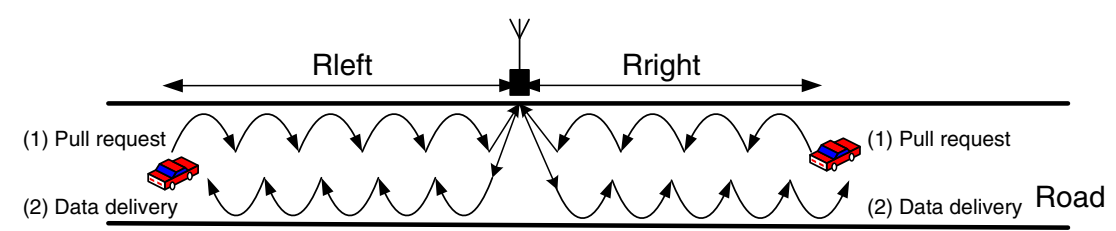

Fig. 2 Coverage of pull. The requesting car (either at left or right of the infostation) first sends out a pull request towards its nearest infostation, forwarded by vehicles in the middle. The infostation then sends the requested data to the vehicle in multi-hops. Note that for both sides, the distance covered by the pull request and the distance covered by the data delivery differ due to mobility where $r_{\text {left }}$ is the distance between the infostation and the leftmost vehicles who obtain the complete broadcast of $i$.

When infostations and vehicles use the same radio configuration, $B_{\text {info }}=B_{\text {mobile }}$ and $R_{\text {info }}=R_{\text {mobile }}$ and the data size is limited, i.e. $\frac{S V}{B_{\text {mobile }} \cdot R_{\text {mobile }}}<<1$, we can reduce $R_{\text {right }}$ as follows:

$R_{\text {right }} \approx \frac{B_{\text {mobile }} \cdot R_{\text {mobile }} \cdot T}{S+S_{\text {pull }}}-\frac{S V}{B_{\text {mobile }}} \frac{S}{S+S_{\text {pull }}}$.

Similarly, we have

$R_{\text {left }} \approx \frac{B_{\text {mobile }} \cdot R_{\text {mobile }} \cdot T}{S+S_{\text {pull }}}+\frac{V S}{B_{\text {mobile }}} \frac{S}{S+S_{\text {pull }}}$.

From the above, we can derive the range of dissemination:

$$
\begin{aligned}
D_{\text {info }}^{\text {pull }} & =R_{\text {right }}+R_{\text {left }} \\
& \approx \frac{2 B_{\text {mobile }} T R_{\text {mobile }}}{S+S_{\text {pull }}}
\end{aligned}
$$

We note that the above density measure only applies to scenarios where in a data lifetime $T$, only one vehicle requests the data. In this case, compared to the push model, the infostation density increases by approximately a factor of $\frac{S+S_{\text {pull }}}{S}$. On the other hand, in order to support multiple $N$ requests in parallel, the bandwidth of $B_{\text {mobile }}$ (and $B_{\text {info }}$ ) could drop by a factor of $f(N)$ where $f(N)$ represents the bandwidth degradation from multi-user contention. This maps to an increase of $f(N)$ in the infostation density.

\subsection{Summary of observations}

The above analysis leads to the following observations:

- Both data size and lifetime are major factors affecting the required infostation density.

- Because the velocity of vehicles is in general much lower than wireless signal propagation speed, it has minimal impact on dissemination performance.
This also means data can be disseminated in the opposite direction of traffic flow.

- Under the same bandwidth/range assumption, the infostation density of pull is always higher than that in the corresponding push scheme.

Though the above simplistic analytical models help us understand the general trends, we expect the actual performance of the dissemination schemes to be quite different in reality. Therefore, in the next three sections we design and evaluate realistic dissemination schemes that can work with commodity WiFi.

\section{Impact of vehicle density and data lifetime}

Our goal is to understand the impact of different properties of vehicular networks on the effectiveness of infostation-based information dissemination. Starting with our analytical models, we gradually relax each of our assumptions and evaluate their impact on our results. We begin with a simple event-driven simulation framework that evaluates the impact of vehicle density and data lifetime on our results.

We implemented an event-driven simulator that incorporates vehicle mobility and transmission delays of data. We simulate a single infostation scenario with uniform density vehicular traffic and implement both push and pull dissemination schemes. For simplicity, we set the size of each data object such that objects can be sent over the air without fragmentation, and examine performance assuming there is only a single object in the network. We also assume no wireless interference, no queuing delays and that all vehicles move in a single line in one direction.

The introduction of vehicles means that each transmission hop can traverse a distance less than the maximum transmission range, based on the positions of nearby vehicles. We consider two scenarios, best case (optimal transmission range per hop) and worst case (worst range per hop). In the best case, there is always a vehicle positioned at exactly the maximum transmission range from the previous hop. Therefore, the effective 
transmission range for intermediate transmissions is $R_{\text {mobile }}$, just as assumed in our analysis. In the worst case, we assume that there is a vehicle just outside the transmission range of the previous hop. Therefore each hop's effective range is decreased by $I_{\text {arrival }} \cdot V$. The corresponding results for the worst case are obtained by replacing $R_{\text {mobile }}$ and $R_{\text {info }}$ in the analytical results with $R_{\text {mobile }}-I_{\text {arrival }} \cdot V$ and $R_{\text {info }}-I_{\text {arrival }} \cdot V$, respectively.

Our simulations vary both data lifetime (between 1 and $30 \mathrm{~s}$ ) and vehicular density through inter-arrival times from 0.2 to $2 \mathrm{~s}$. We set vehicle speed $V$ to $30 \mathrm{~m} / \mathrm{s}$, such that an inter-arrival time of $x$ seconds corresponds to a distance of $30 \times \mathrm{m}$ between consecutive vehicles. Furthermore, we assume bandwidth at both infostation and vehicles ( $B_{\text {info }}, B_{\text {mobile }}$ ) is 5 Mbps, max transmission ranges for all radios ( $\left.R_{\text {info }}, R_{\text {mobile }}\right)$ to be $100 \mathrm{~m}$, and objects are $1 \mathrm{Mb}$ in size $\left(S=S_{\text {pull }}=1 \mathrm{Mb}\right)$. Finally, each result is calculated as the mean of 20 simulation runs.

The push scheme We begin by measuring the dissemination range as vehicular density varies. We show two representative plots. Figure 3 explores push performance, both varying data lifetimes with a fixed intervehicle distance of $60 \mathrm{~m}$, and varying vehicle density with a fixed data lifetime of $30 \mathrm{~s}$. Our simulated dissemination ranges fall between the best and worst case models, thus validating our analytical results. In fact, for uniform distribution, we can predict the exact dissemination range by observing that the effective range for intermediate transmissions is $I_{\text {arrival }} \cdot V\left\lfloor\frac{R_{\text {mobile }}}{I_{\text {arrival }} \cdot V}\right\rfloor$. Figure 3a plots the dissemination range for $R=100$ $\mathrm{m}$ and $I_{\text {arrival }}$ between 0.2 and $2 \mathrm{~s}$. Figure $3 \mathrm{~b}$ shows that for a fixed vehicle density, the expected dissemination range increases linearly with the lifetime of the

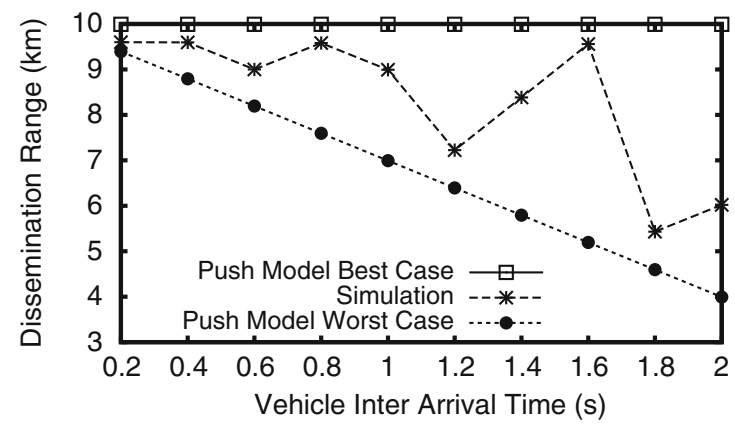

(a) Data Lifetime $10 \mathrm{sec}$

Fig. 3 The push scheme dissemination range in the event simulation. a The dissemination range is bound by the best and worst case models, and can be predicted by the effective transmission data. This follows directly from the analytical models described in the previous section.

The pull scheme For this simulation, we allow all the vehicles along the length of the road to issue pull requests and measure the farthest vehicles that successfully receive responses back from the info station. Since the event simulation ignores interference and queuing delays, each packet pair (pull request and the corresponding data) in the network is independent of other transmissions (note: we later repeat this experiment with realistic interference in our QualNet simulations). Like the push case, the dissemination ranges are bound by the best and worst case ranges predicted by our model. We also observe that the dissemination range is approximately half of that observed in the corresponding push scheme. Since pull requests are equal in size to response packets, approximately half of the round-trip lifetime is used to deliver the request to the info station and the other half is used to deliver the response to the requester.

For both the push and pull schemes, since the velocity of vehicles is much lower than wireless signal propagation speed, it has minimal impact on dissemination performance.

\section{Making it work in practice}

Both dissemination models rely on multi-hop data dissemination to cover a large number of vehicles. Effective multi-hop data dissemination in real world systems, however, faces several challenges, including wireless interference, unreliable transmissions, and queuing delays at nodes. In this section, we describe several challenges faced in a realistic wireless setting,

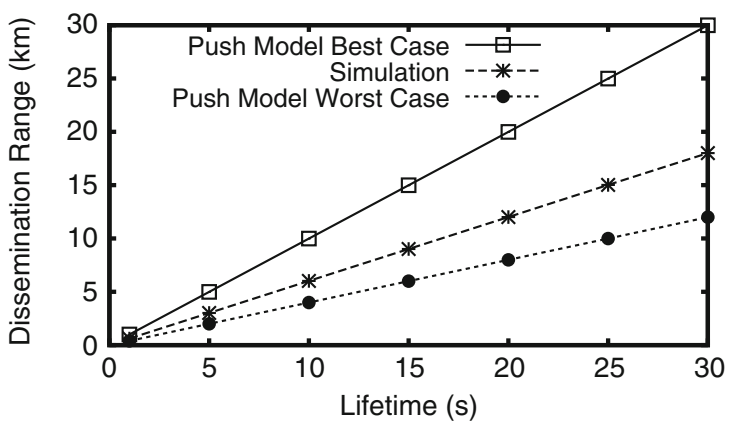

(b) Inter Arrival Time $2 \mathrm{sec}$

range $\left(I_{\text {arrival }} \cdot V\left\lfloor\frac{R_{\text {mobile }}}{I_{\text {arrival }} \cdot V}\right\rfloor\right)$. b Given a fixed vehicular density, dissemination range increases linearly with data lifetime 
and the techniques we proposed to address them and improve transmission bandwidth/range. Because our focus is not on detailed performance optimizations, we focus only on high level techniques that have significant impact on the overall required density of infostations. Some of the proposed techniques are inspired by existing works, and our contribution is to integrate necessary techniques together to enable a reasonable evaluation of the infostation density.

\subsection{Position-guided group broadcast}

Our first challenge is how to reliably transmit data between two moving vehicles or a vehicle and a nearby infostation. We choose a group-based broadcast model for data transmission rather than a point-to-point routing approach. There are two reasons for this design decision. First, the high rates of mobility in a vehicular network prevent efficient discovery and maintenance of multi-hop routes. Traditional techniques such as AODV [18] or DSR [8] would require frequent network-wide broadcasts as routes quickly degrade and are restored. Second, infostations will often disseminate highly popular data desired by the majority of nearby vehicles, e.g. real-time traffic conditions. Such communication naturally lends itself to a broadcast communication model.

Traditional MAC-level broadcasts are clearly insufficient for our application scenario. Network-wide broadcasts would incur extremely high per-message overhead, and uncoordinated broadcast messages would result in significant contention and packet loss. Since there are no reliability mechanisms at the MAC layer, data dissemination would be highly unreliable and data would have extremely limited transmission ranges, thus requiring artificially high density of infostations for effective network coverage.

Position-guided group broadcast We propose a position-based broadcast that exploits the unidimensional nature of vehicular traffic. We assume that wirelessly-connected vehicles can obtain their geographic locations either from widely available GPS devices or through wireless triangulation on cellular networks. A node determines the data dissemination direction based on its relative location to the message source, and broadcasts the message. When a node receives a packet from a neighbor (previous hop), it starts a propagation timer whose expiration time is linearly proportional to the number of cars between itself and the maximum transmission range of the previous hop. If it does not hear any retransmissions of this message before its timer expires, it retransmits

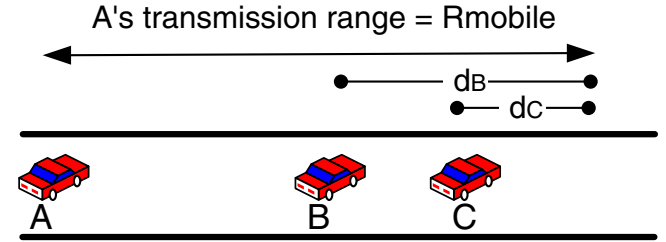

Fig. 4 Position-guided group broadcast. When vehicles $B$ and $C$ receive $A$ 's broadcast of data $i$, both initiate a local timer that is proportional to their distance to the edge of $A$ 's transmission range, $d_{B}$ and $d_{C}$

the data forward. The node cancels its timer if it hears someone else's retransmission ${ }^{2}$ before the timer expires. We illustrate the process in Fig. 4, where vehicle $C$ 's propagation timer goes off first, since it is the vehicle closest to the edge of the transmission range. Intuitively, this allows the nodes farthest from the previous hop to retransmit first while suppressing redundant messages from closer nodes. This can be seen as a form of directional gossip, and is similar in principle to techniques used in the Trickle code dissemination system [11], ExOR [3] and [4, 7, 16].

Limitations While generally effective, this directional gossip method has limitations in practice. First, retransmission at each hop relies on the nodes' ability to receive transmissions from nearby neighbors, and suppressing their own transmissions to reduce interference. However, a node cannot always predict the actions of a neighbor farther down the dissemination path using the propagation timer. For example, wireless interference at a farther node might force it to back off its transmission. But an intermediate node might incorrectly assume that no farther node has received the packet and retransmit the packet itself when its timer expires. This results in redundant transmissions and interference. This is a form of the hidden terminal problem, and cannot be addressed using explicit acknowledgments because they themselves can be lost to interference.

Second, our technique requires each node to know the approximate positions of its neighbors, in order to estimate the value for its propagation timer. While this is trivial with uniform distribution of vehicles, it is a challenge for real world scenarios. In practice, we expect nodes to periodically broadcast their location and velocity data to their neighbors. Such data is also required to support widely-proposed traffic safety applications, and can either be broadcasted on a separate

\footnotetext{
${ }^{2}$ Only transmissions from nodes closer to the destination than the current node cancel its propagation timer.
} 
control channel or embedded into regular data packets. For our experiments, we evaluate two schemes, an $\mathrm{Or}$ acle scheme where nodes know the relative positions of other nodes in their neighborhood, and a Naive scheme where each node predicts the positions of its neighbors based on an estimate of vehicle density in the entire network.

\subsection{Eliminating redundant traffic}

Minimizing wireless interference is crucial to improving performance of our data dissemination framework. This is especially relevant for traffic in the pull and hybrid dissemination schemes, where multiple pull requests must contend with each other and reply data for available bandwidth. Therefore, we must eliminate excess traffic when possible. First, each message (pull request, pull reply or push message) has an inherent direction. We embed the source and destination addresses and locations inside packet headers to suppress forwarding in the opposite direction. Second, since pull requests and responses have the infostation or requester as a destination, once a pull message reaches its destination (estimated by the destination location in the header), further transmissions are dropped. This destination-awareness helps to minimize unnecessary network traffic, thus also reducing congestion and interference.

\subsection{Decoupling control with explicit probes}

Our position-guided broadcasts implicitly use data packets for media contention, i.e. nodes listen for neighbor transmissions to determine if they need to forward the data. However, this leads to performance issues in the presence of large data packets. Large packets take longer to transmit and receive, especially if fragmentation and reassembly occur at the wireless MAC layer. Because nodes are only notified of a message when the entire message has been received, timers can expire at nodes listening for neighbor transmissions because a neighbor has not finished transmitting a large message. This results in multiple nodes transmitting the same data and causing interference. The presence of variable sized messages means that propagation timers are often inaccurate, either set too low, expiring before larger messages could be received or set too high, unnecessarily reducing propagation performance.

We address this issue by decoupling data messages from channel contention messages. We introduce an explicit probe message that is broadcast by a node to indicate its desire to forward the data packet. The probe packet is small, but includes the hash of its associated data packet. Neighbors receiving this packet understand a neighbor is about to transmit, and cancel their own timers. Because these probes are small and fixed in size, we can accurately set propagation timers to account for their transmission time.

\subsection{Reducing collisions}

In the pull model, there is high probability of collision between the small pull requests and the large data packets traveling in opposite directions. To minimize these collisions, nodes receiving the probe packets set a busy time stamp and avoid transmitting any packets as long as this time stamp is valid. The time stamp is valid for the duration of the ongoing data packet transmission. Additionally, every node stores the pull requests it has forwarded in its local buffer. A pull packet is dropped from the buffer of a node if it gets an indirect ack from another node closer to the infostation or the buffer timer expires, whichever comes first. When a node receives a data packet, it forwards any buffered pull packets after giving enough time for the data packet to pass by. The idea is to retransmit those pull packets that might have collided with an oncoming data packet transmission.

While this pull buffering technique improves the performance of pull dissemination, it does not achieve optimal results in a real multi-hop network. This is because the interference and backoff timers at the MAC layer [2] may lead a node to make incorrect assumptions about the transmissions in the neighborhood. For example, a node receiving a long data transmission might still experience interference from a nearby pull packet transmission though its busy time stamp is set. Our simulations in Section 6.2 quantify this effect.

\section{QualNet simulations and results}

We perform realistic evaluation of the dissemination schemes through detailed QualNet simulations that take into account real MAC protocols, wireless interference, queuing delays and propagation effects. Using techniques described in the previous section, we implemented the push and pull schemes as application-level modules in QualNet. We use Constant Bit Rate (CBR) traffic on top of an 802.11 MAC layer. We hope to use these experiments to answer three key questions. (1) Do our techniques perform well on realistic vehicular traffic patterns? (2) Can we improve dissemination reliability by increasing infostation deployment density? (3) How is dissemination performance affected 


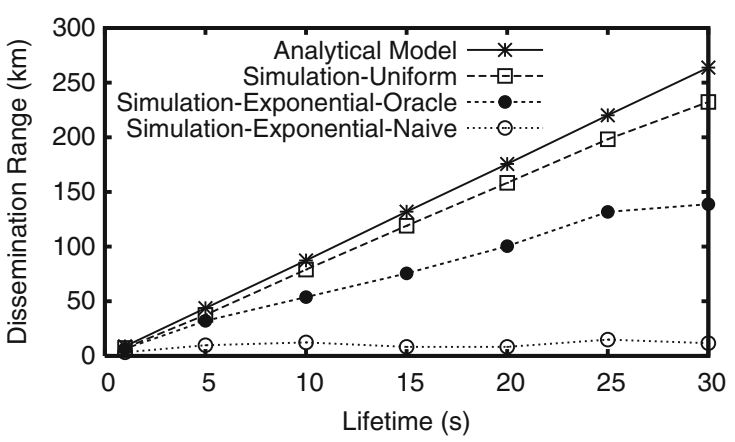

(a) The Push Scheme

Fig. 5 Dissemination range (controlled traces). The results show that uniform distribution performs better than exponential because the effective transmission range at each hop is likely larger for uniform. Naive performs badly compared to Oracle

by incomplete adoption of our system (less than 100\% penetration ratio)?

We perform our experiments on three separate vehicle traces:

Uniform Vehicles are uniformly distributed with a spacing of $60 \mathrm{~m}$ between consecutive vehicles and a uniform constant velocity of $30 \mathrm{~m} / \mathrm{s}(\approx 67 \mathrm{mi} / \mathrm{hr})$.

Exponential We introduce vehicles into the network with distance between consecutive vehicles defined as an exponential distribution bounded by the vehicle transmission range. Average distance between consecutive vehicles is $60 \mathrm{~m}$ and vehicles travel at a uniform constant velocity of $30 \mathrm{~m} / \mathrm{s}$. Bounding the inter-vehicle distance minimizes the probability of disconnection in the network.

Real measurements We downloaded a publicly available highway vehicle trace from NGSIM [17]. This trace consists of data collected on a 2,100 ft segment of the five-lane Southbound US-101 highway over a period of 45 min on June 252005 . Vehicle density in this trace is high, with an average distance of $20 \mathrm{~m}$ between consecutive vehicles in the same lane. Since the trace itself does not have sufficient data points, we build a complex 21-state Markov model using statistics from the trace, then use the model to generate arbitrarily long synthetic traces while preserving the statistical properties of the original trace. Details on the trace modeling and synthesis are beyond the scope of this paper. We set both transmission ranges of infostations and vehicles ( $\left.R_{\text {info }}, R_{\text {mobile }}\right)$ to $485 \mathrm{~m}$, transmission bandwidth for both infostations and vehicles $\left(B_{\text {info }}, B_{\text {mobile }}\right)$ to $1 \mathrm{Mbps}$, size of all data objects $(S)$ to 12,500 Bytes, and size of pull requests $\left(S_{\text {pull }}\right)$ to 200 Bytes. Applying these parameters into the analytical results of Section 3, we

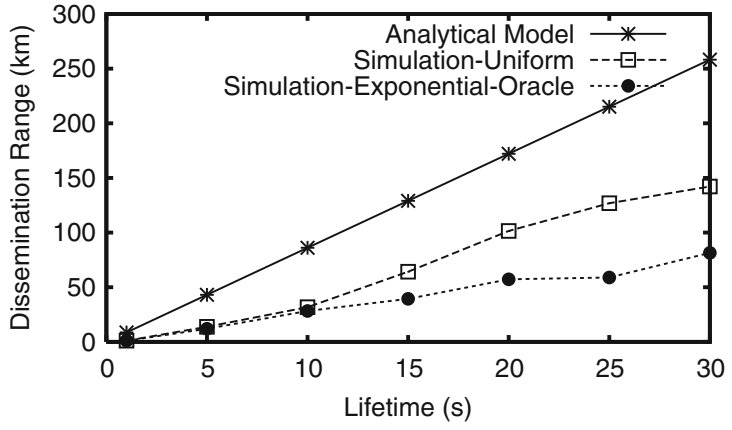

(b) The Pull Scheme

due to the contention from redundant transmissions resulting from inaccurate propagation timers. The pull scheme has lower dissemination range than the push scheme due to contention among pull requests

derive the theoretical infostation density (marked as "analytical model"), which represents the upper bound on the performance.

\subsection{Performance of the push and pull schemes}

Our key metrics in the evaluation are the effective dissemination range, which determines the necessary infostation density, and the reception percentage, ratio of vehicles in the dissemination range that receive their desired data.

Dissemination range In these experiments we measure the dissemination ranges of push and pull as data lifetime increases. From Fig. 5a, we note that both Uniform and Exponential with Oracle perform well, while the Exponential-Naive suffers heavy degradation. The poor performance of Exponential-Naive can be attributed to the fact that, without location information of nearby vehicles, vehicles' propagation timers can be highly inaccurate, resulting in redundant transmissions of the same data. The ensuing contention and interference cause data collisions and propagation delays, resulting in low dissemination ranges. Uniform performs better than Exponential with Oracle because the effective transmission range at each intermediate hop is likely to be higher for uniform than exponential distribution.

In the pull scheme, increasing data lifetimes also increases the segment of the road that an infostation is responsible for, thus increasing the number of incoming pull request. ${ }^{3}$ Figure $5 \mathrm{~b}$ shows that pull follows the

\footnotetext{
${ }^{3}$ Unless specified otherwise, the number of pull requests is proportional to the length of the road ( $1 \mathrm{request} / 2 \mathrm{~km}$ ) in all our pull experiments.
} 


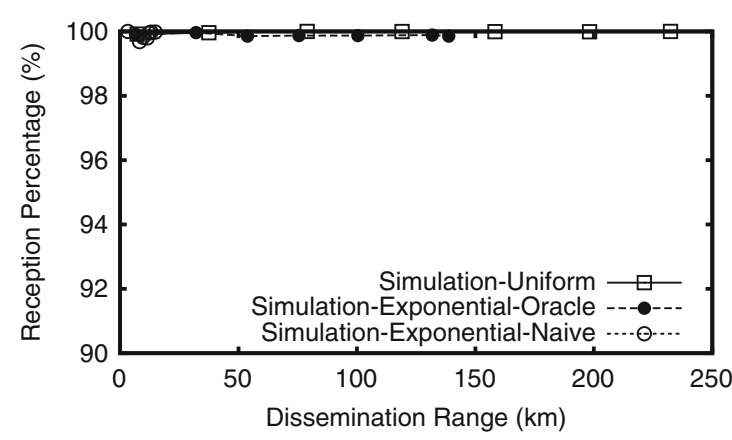

(a) The Push Scheme

Fig. 6 Reception percentage (controlled traces). In the push scheme nearly $100 \%$ of the expected cars get their data. In the pull scheme, the reception percentage decreases with increase in

same trend as the push scheme albeit with lower values for dissemination ranges. This is because our analytical model for pull assumes no wireless interference between different pull requests, but in reality wireless interference heavily affects the performance. This interference lowers effective bandwidth in the system, and contention leads to increased transmission delays. In contrast, the push scheme has minimum interference since there is no cross traffic at any point in time.

Reception percentage Since dissemination range is calculated based on the farthest car reached by data from the infostation, it does not quantify the number of vehicles in the range that actually receive their data. To quantify this, we calculate the reception percentage, the percentage of the vehicles expecting data that receive their data. In the push model, all vehicles in the dissemination range expect data. In the pull model, only those that sent requests expect data.

Figure 6a plots the reception percentage for the push experiment we did in Fig. 5a. We can see that the reception percentage is nearly $100 \%$, thus validating

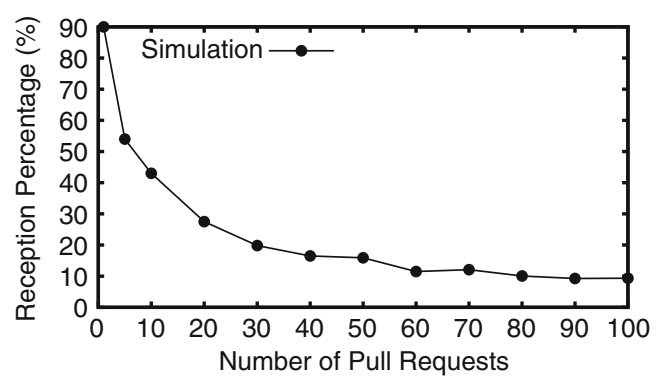

Fig. 7 Effect of varying pull requests (uniform trace). The reception percentage drops exponentially as number of requests increases. This results from increased contention between the pull requests and responses, leading to collisions and large delays

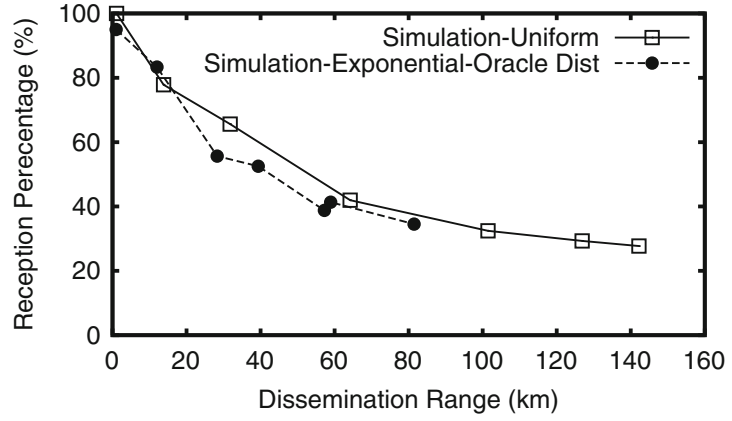

(b) The Pull Scheme

the length of road because the number of requests contending for the available bandwidth increases leading to interference and collisions

the effectiveness of the dissemination techniques. For the pull scheme, we generate requests from the observed dissemination ranges of Fig. 5b. We see from Fig. $6 \mathrm{~b}$ that the reception percentage decreases with longer segments of the road. Since pull request density per road length is constant, longer roads mean more incoming requests and increased contention.

To investigate the impact of pull requests further, we ran a simulation varying the number of pull requests in the network. Specifically, we fix the lifetime of the data to $30 \mathrm{~s}$ and vary the number of pull requests from 1 to 100 on a fixed length of road. Figure 7 shows that the reception percentage decreases exponentially as the number of requests increases. This is from increased requests contending for the same bandwidth leading to collisions and large delays.

\subsection{Impact of dissemination optimizations}

To quantify the effectiveness of our optimizations discussed in Section 5, we measure the impact of destination-awareness and pull buffering techniques

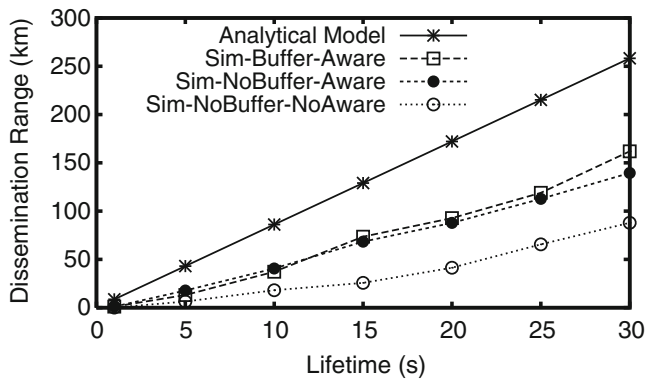

Fig. 8 Performance of various dissemination techniques for the pull scheme (uniform trace). Buffer and destination awareness improve the performance of dissemination by reducing congestion and retransmitting potentially collided pull requests 


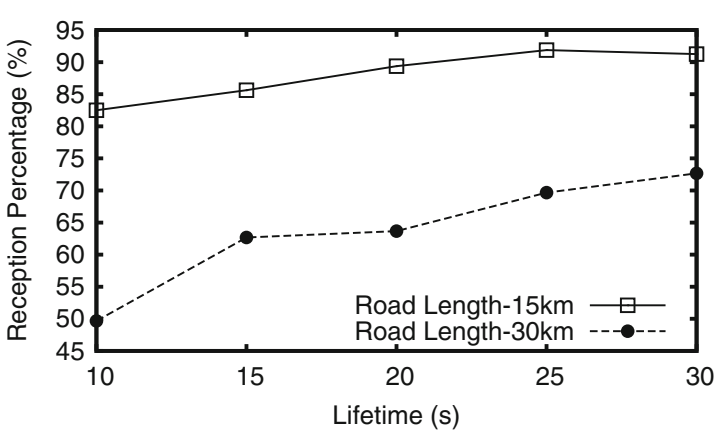

(a) Increasing Lifetime

Fig. 9 The pull scheme (exponential trace). Reception percentage with increasing lifetime and increasing infostation density. a One can improve the reception percentage by increasing the lifetime because the data has more time to reach the destination.

on dissemination ranges in the pull scheme. Vehicles are uniformly distributed. Our results on pull scheme (Fig. 8) show that as expected, utilizing both techniques achieves the best dissemination performance. While destination-awareness reduces unnecessary traffic, pull buffer retransmits potentially collided pull requests allowing them to reach the infostation. The highest gain was achieved by reducing traffic using destinationawareness. In comparison, pull buffering produced less benefits in the presence of wireless interference. A node that did not receive an (indirect) acknowledgment cannot be sure if a request is lost or if the sender backed off due to interference, and might incorrectly retransmit causing congestion.

\subsection{Improving pull performance}

Our earlier results show that reception percentage is poor for pull scenarios with multiple pull requests. We

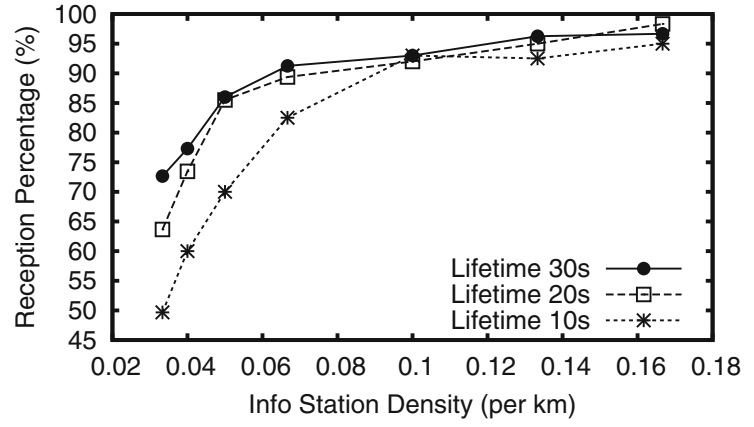

(b) Increasing Info Station Density

Orthogonally, b shows that as info station density increases reception percentage improves because there are fewer requests contending for an infostation and hence less delay and collisions

propose two solutions to improve the reception percentage. First, we can increase the lifetime of the data for a given dissemination range, so that data packets have more time to reach their destinations. We experiment with two traces: (1) a $30 \mathrm{~km}$ exponential trace which corresponds to $10 \mathrm{~s}$ lifetime (from Fig. 5b), and (2) a $15 \mathrm{~km}$ exponential trace with $10 \mathrm{~s}$ lifetime, which essentially doubles the infostation density. We see from Fig. 9a that by increasing data lifetime, we have improved reception percentage from $50 \%$ to about $73 \%$ for the $30 \mathrm{~km}$ trace and $82-92 \%$ for the $15 \mathrm{~km}$ trace.

Alternatively, by increasing the infostation density, the number of pull requests contending for service from any single info station can be reduced. The reduction in interference will lead to an improved reception percentage. To validate this, we experiment with data of lifetimes $10 \mathrm{~s}, 20 \mathrm{~s}$ and $30 \mathrm{~s}$ and vary the distance between consecutive infostations from $30 \mathrm{~km}$ to $5 \mathrm{~km}$. The vehicles are exponentially distributed. Figure $9 \mathrm{~b}$

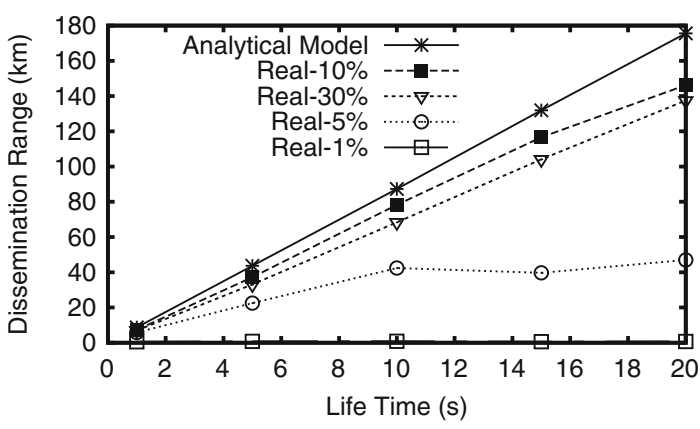

(a) Impact of Penetration Ratio with varying Lifetime

Fig. 10 The push scheme (real trace): Impact of penetration ratio. As the penetration ratio increases from $1-10 \%$ dissemination performance improves because of increased connectivity. Further

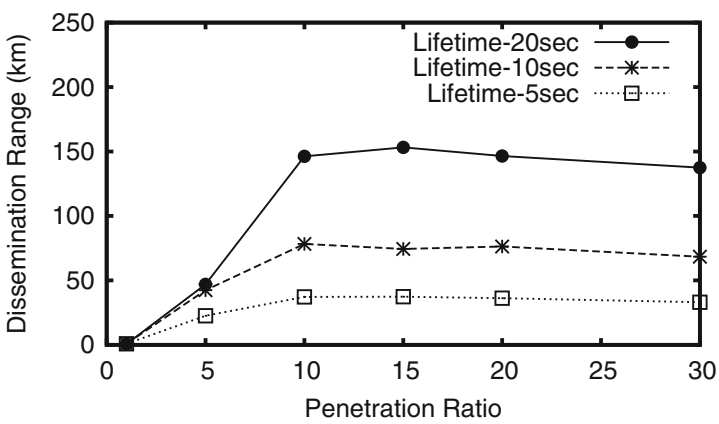

(b) Impact of Life time with varying Penetration Ratio

increase in penetration ratio leads to a slight decrease in the performance because of transmission delays due to contention between vehicles 


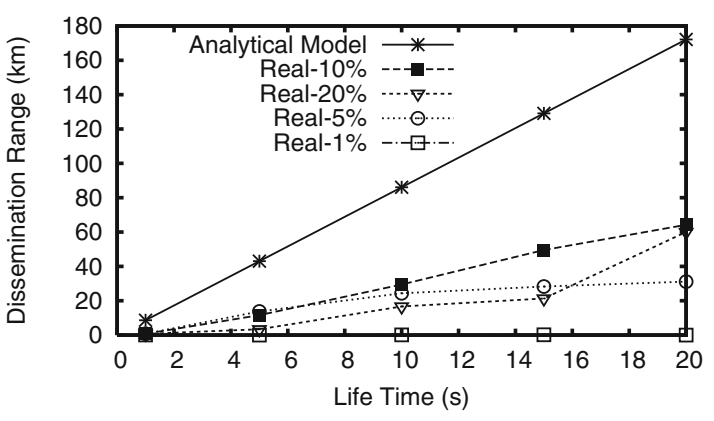

(a) Impact of Penetration Ratio with varying Lifetime

Fig. 11 The pull scheme (real trace). Impact of penetration ratio. Dissemination range increases as penetration ratio increases from $1-10 \%$. Further increase in penetration ratio leads to in-

shows that reception percentages for all three lifetimes increase with increase in infostation density.

\subsection{Impact of penetration ratio}

As vehicular networks are initially deployed, the number of vehicles equipped with wireless devices that participate in a particular network will be low. This penetration ratio (active participants as a ratio of all vehicles on the road) plays a crucial part in the performance of dissemination schemes. To understand its impact, we randomly equip $\mathrm{x} \%$ of vehicles in our real trace with wireless radios and observe the impact on dissemination ranges of the push and pull schemes.

Figure 10a plots the push dissemination range of various penetration ratios with increasing lifetime. When the penetration ratio is $5 \%$ or less, disconnections in the network lead to low dissemination ranges. With $10 \%$ penetration ratio, there are enough participant vehicles to maintain network connectivity, thereby producing dissemination ranges similar to those predicted by our model. Interestingly, with $30 \%$ penetration ratio the dissemination range slightly decreases compared to $10 \%$. This is because an increasing number of participants starts to produce contention among neighbors at each hop, leading to transmission delays. Similarly, when we vary the penetration ratio (Fig. 10b), we observe that dissemination performance drops after $10 \%$ penetration.

Similarly for the pull scenario, Figure 11 shows that the optimal dissemination performance can be observed at a penetration ratio of $10 \%$. Further increase in penetration ratio leads to a decrease in the dissemination range due to increased contention. Figure $11 \mathrm{~b}$ shows that for all the three lifetimes, the dissemination performance increases as penetration ratio increases from $1-10 \%$, but gradually starts decreasing with a further increase in the penetration ratio.

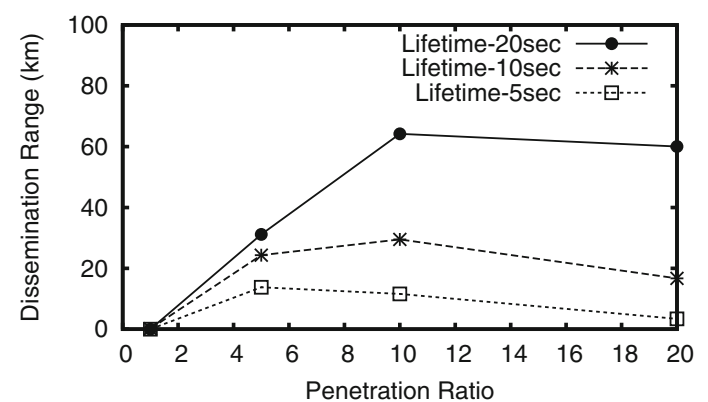

(b) Impact of Lifetime with varying Penetration Ratio

creased contention among the equipped vehicles which results in a decrease in the dissemination performance

\section{Conclusion}

To evaluate the feasibility of infostation-based data dissemination, we seek to answer two key questions. Given an application's requirements for data lifetime, how densely must we deploy infostations, and what fraction of the vehicles need to be participants in our network? Our study shows that dissemination using the push model provides the best results. With a penetration ratio of only $10 \%$, the system can support data with lifetime of $15 \mathrm{~s}$ or longer using infostations spaced $100 \mathrm{~km}$ apart. For the same scenario, the pull model requires a much higher density as expected, with infostations spaced approximately every $10-20 \mathrm{~km}$. Finally, our results support the hypothesis that vehicleto-vehicle distribution combined with intermittent connectivity to infostations does indeed provide a suitable data dissemination system for a vehicular network, at least in highway scenarios.

Open Access This article is distributed under the terms of the Creative Commons Attribution Noncommercial License which permits any noncommercial use, distribution, and reproduction in any medium, provided the original author(s) and source are credited.

\section{References}

1. Balasubramanian A et al (2007) Web search from a bus. In: CHANTS

2. Bianchi G (2000) Performance analysis of the IEEE 802.11 distributed coordination function. IEEE JSAC 18:3

3. Biswas S, Morris R (2005) Exor: opportunistic multi-hop routing for wireless networks. In: Proc. of SIGCOMM

4. Briesemeister L, Schafers L, Hommel G (2000) Disseminating messages among highly mobile hosts based on intervehicle communication. In: Proc. IEEE IV

5. Bychkovsky V et al (2006) A measurement study of vehicular internet access using unplanned 802.11 networks. In: Proc. of MobiCom 
6. Chen ZD, Kung HT, Vlah D (2001) Ad hoc relay wireless networks over moving vehicles on highways. In: Proc. of MobiHoc

7. Jeong J, Guo S, Gu Y, He T, Du D (2010) Trajectorybased data forwarding for light-traffic vehicular ad-hoc networks. In: Proc. of IEEE Transactions on Parallel Distributed Systems

8. Johnson D, Maltz D (2001) Dynamic source routing in ad hoc wireless networks. In: Ad hoc networking

9. Lee K et al (2007) First experience with cartorrent in a real vehicular ad hoc network testbed. In: Proc. of MOVE

10. Lee U et al (2006) Fleanet: a virtual market place on vehicular networks. In: Proc. of V2VCOM

11. Levis P, Patel N, Culler D, Shenker S (2004) Trickle: a selfregulating algorithm for code propagation and maintenance in wireless sensor networks. In: Proc. of NSDI

12. Little T, Agarwal A (2005) An information propagation scheme for vanets. In: IEEE ITSC

13. Lochert C, Scheuermann B, Caliskan M, Mauve M (2007) The feasibility of information dissemination in vehicular adhoc networks. In: Proc. of WONS

14. Mahajan R, Zahorjan J, Zill B (2007) Understanding wifi-based connectivity from moving vehicle. In: Proc. of IMC
15. Nadeem T, Shankar P, Iftode L (2006) Comparative study of data dissemination models for vanets. In: Proc. of mobiquitous

16. Nath B, Niculescu D (2003) Routing on a curve. ACM Comput Commun Rev 33(1):160

17. NGSIM HomePage. http://ngsim.fhwa.dot.gov

18. Perkins C, Royer E (1999) Ad hoc on-demand distance vector routing. In: Proc. of WMCSA

19. QualNet. http://scalable-networks.com

20. Skordylis A, Trigoni N (2008) Delay-bounded routing in vehicular ad-hoc networks. In: Proc. of MobiHoc

21. Torrent-Moreno M (2007) Inter-vehicle communications: assessing information dissemination under safety constraints. In: Proc. of WONS

22. Wischhof L, Ebner A, Rohling H (2005) Information dissemination in self-organizing intervehicle networks. IEEE Trans ITS 6(1):90-101

23. Zhang X et al (2007) Study of a bus-based disruption tolerant network: mobility modeling and impact on routing. In: Proc. of MobiCom

24. Zhao J, Cao G (2006) Vadd: vehicle-assisted data delivery in vehicular ad hoc networks. In: IEEE INFOCOM

25. Zhao J, Zhang Y, Cao G (2007) Data pouring and buffering on the road: a new data dissemination paradigm for vehicular ad hoc networks. In: IEEE trans. on VT 